\title{
The Application of the " $3+1$ " Mode in the COVID-19 Epidemic Prevention and Control at the Infection Ward of a Designated Comprehensive Hospital for COVID-19 Treatment
}

\author{
Lihua Zheng ${ }^{\circledR}$, Beixi Wu, Hongmei Pan, Xiumei Zhong, Qiong Shu, Haotong Xu, Shuxian Jiang* \\ Department of Infectious Diseases, The Third Affiliated Hospital of Sun Yat-sen University, Guangzhou, Guangdong \\ Email: ^2390059691@qq.com
}

How to cite this paper: Zheng, L.H., Wu, B.X., Pan, H.M., Zhong, X.M., Shu, Q., Xu, H.T. and Jiang, S.X. (2021) The Application of the " $3+1$ " Mode in the COVID-19 Epidemic Prevention and Control at the Infection Ward of a Designated Comprehensive Hospital for COVID-19 Treatment. Open Journal of Nursing, 11, 489-496. https://doi.org/10.4236/ojn.2021.116042

Received: May 12, 2021

Accepted: June 21, 2021

Published: June 24, 2021

Copyright $\odot 2021$ by author(s) and Scientific Research Publishing Inc. This work is licensed under the Creative Commons Attribution International License (CC BY 4.0).

http://creativecommons.org/licenses/by/4.0/

\begin{abstract}
Objective: We assess the application effect of the " $3+1$ " mode in the COVID-19 epidemic prevention and control at the infection ward of a designated comprehensive hospital for COVID-19 treatment. Method: Based on the features of the inpatients of the infection ward and their relatives, a " 3 +1 " mode for the COVID-19 prevention and control is developed to conform to the demands for epidemic prevention and control and the overall prevention and control scheme of the whole hospital. Here, "3" stands for the epidemic prevention and control system, personnel management, and prevention and control measures; "1" stands for COVID-19 testing. Result: From March 1, 2020 to March 31, 2021, a total of 3056 patients were hospitalized in the three infection wards. Among them, 265 patients had a fever, and 113 patients had respiratory symptoms. None of them were infected with COVID-19. The participation rate of the test about the knowledge related to COVID-19 and the knowledge mastery rate were both $100 \%$ among the medical staff. None of the inpatients and their companions or the medical staff was diagnosed with COVID-19. Conclusion: Thus the " $3+1$ " mode proves successful for avoiding nosocomial infection and the spread of the epidemic.
\end{abstract}

\section{Keywords}

COVID-19, Comprehensive Hospital, Infection Department, Epidemic Prevention and Control 


\section{Introduction}

During the COVID-19 epidemic, there has been an urgent need to normalize COVID-19 prevention and control work, identify potential risks, avoid epidemic spread and nosocomial infection, protect the health and safety of medical staff, inpatients and their companions, and ensure the quality and safety of healthcare services. To achieve the above goals, Guangzhou brings into full play the advantages and potentials of the joint conference system for comprehensive reform of public hospitals. Medical resources of different levels and different categories, which form a pyramid-shaped network of healthcare services, are subject to unified allocation. Nine provincial- or municipal-level hospitals, 12 district-level hospitals, 45 district-level designated hospitals, and 104 fever clinics are designated for COVID-19 treatment in Guangdong Province. A municipal-level unified coordination mechanism for referral and consultation is established. A treatment chain is thus formed. A closed-loop management model consisting of primary screening, district-level observation, and municipal-level admission and treatment is built [1]. Our hospital is a designated comprehensive hospital for COVID-19 treatment in Guangdong Province. The infection department has a large number of patients with a complex disease composition. The patient turnover and population mobility are high at the infection department. Thus, the infection department is a weak and important site for COVID-19 prevention and control, which dictates normalizing the COVID-19 prevention and control.

\section{Subjects and Method}

\subsection{Subjects}

Subjects were recruited among patients admitted to the infection ward from March 1, 2020 to March 31, 2021 and their companions. The subjects were excluded if any of the following exclusion criteria was met according to the diagnostic criteria specified in the COVID-19 Diagnosis and Treatment Protocol (6th Edition): 1) Having fever $\left(\mathrm{T}>37.3^{\circ} \mathrm{C}\right.$ ) and (or) respiratory tract symptoms; 2) Having a positive epidemiological history for COVID-19; 3) Normal or reduced total white blood cell count and lymphocyte count; 4) Having positive radiological manifestations upon chest CT [2]. All of the enrolled subjects were required to provide proof of a negative result of the PCR COVID-19 test valid for 7 days. The patients and their companions were also asked to receive routine blood test, chest CT and COVID-19 antibody testing if necessary. COVID-19 could not be easily precluded in personnel coming from high- and intermediate-risk areas despite the proof of a negative result of the PCR COVID-19 test. Admission was not allowed unless the result was negative upon repeated nucleic acid tests.

\subsection{Method}

Based on the features of the inpatients of the infection ward and their relatives, a " $3+1$ " mode for the COVID-19 prevention and control is developed to conform 
to the demands for epidemic prevention and control and the overall prevention and control scheme of the whole hospital. Here, " 3 " stands for perfecting the epidemic prevention and control system, enhancing the administration of patients and medical staff, and putting the prevention and control measures into effect; "1" stands for COVID-19 testing [3] [4] [5] [6].

\subsubsection{Perfecting the Epidemic Prevention and Control System}

The epidemic prevention and control system is timely adapted and modified. The emergency response plan is constantly perfected, and drills are carried out. The working system and workflow guidance for COVID-19 prevention and control at the infection department are timely revised, updated, reviewed, and filed with the hospital infection control department according to the latest epidemic prevention and control guidelines from the superiors and the distinctive features and practice of the infection department. Our infection department has developed the emergency response plan adapted to different situations with enhanced visions and initiative. The workflow measures of each link are refined, and the specific responsibilities are clearly defined for each personnel and duty. Desktop maneuvers and field exercises are carried out to identify loopholes and defects. Continuous efforts are made to optimize the emergency response plan and improve the actual combat capacity. The hospital organizes drills jointly attended by different functional departments, including the medical affairs department, nursing department, hospital infection-control department, logistics department, quality management department and equipment department. The responsibilities of each functional department are laid down, the workflow is straightened out, and the linkage response mechanism for the COVID-19 epidemic is established.

\subsubsection{Enhancing Personnel Management}

1) Strengthening the differentiation of suspected patients. The infectious disease clinic conducts regular training sessions on epidemic control and prevention and pre-examination and triage process. The physicians are educated on the importance of being alert and watchful to COVID-19 screening during consultation hours. The physicians at the infection department are informed of the common symptoms of COVID-19. Standard quarantine or measures against the epidemic spread is implemented once a patient is highly suspected of COVID-19. Precautions are also taken for those accompanying the suspected cases. The probability of COVID-19 cannot be easily precluded in patients with an unfavorable epidemiological history. Repeated screening tests should be administered to these patients. Precautions should be strictly taken in every link before it is $100 \%$ sure that the patient is not infected with COVID-19.

2) Tightening the administration of visitors and companions at the ward

a) Patient visiting at the ward is subject to strict control and supervision. Patient visiting at the ward is forbidden in principle. Ward access is shut down 24 hours a day, with specialized personnel in attendance. Outsiders are kept out of the ward and not allowed to enter the ward. Under special situations (eg., disease 
change and need for diagnostic and therapeutic procedures) or in case of the need to go through the discharge proceedings, the following precautions are strictly taken for anyone waiting to enter the ward: check the health code (green or blue code), measure the body temperature, instruct on how to wear the mask correctly, inquire of the epidemiological history, and sign the commitment letter. One or two people are allowed for each visit for no longer than $30 \mathrm{~min}$. Any visitors of the ward should be carefully registered. The time of coming and out of the ward should be recorded (exact to the minute). The visitors are not allowed to enter other wards or aggregate in groups [6].

b) Accompanying the patients at the ward is strictly managed. No person is allowed to stay in the ward to accompany the inpatients. If there is indeed a need for a companion due to the disease, the person that stays should receive nucleic acid tests and sample collection. After precluding the possibility of COVID-19, the companions should go through relevant proceedings at the ward where the patient is in, carrying the proof of a negative result of the PCR COVID-19 test. The proof is valid within 7 days since its issue. The results of the nucleic acid test presented by other hospitals are valid within 7 days. One companion is allowed for one patient and is required to wear a wristband. The doctor provides instructions for the companions. One companion is allowed for one bed. Multiple or alternate companions for one bed is forbidden. The companions are inquired of the epidemiological history, and their body temperature is taken. The registration form for companions in the ward is filled. The companions are required to sign the commitment letter for ward visitors and companions. The wristband is distributed among the companions as proof, on which the bed No., companion's name, and companion's ID number are written. It is ensured that the companion's information on the wristband is correct. The companion's wristband is differentiated from the patient's wristband in color. If there is a need to replace the previous companion with a new one under special situations, the new companion must receive a nucleic acid test and go through relevant proceedings. The wristband is retrieved from the previous companion and destroyed at the ward. The body temperature of the companions is taken twice daily and registered. The companion's ID is checked once daily to ensure consistency between the person and the ID. The food is distributed to patients and companions by the hospital canteen for patients. The patients or the companions cannot order food outside the hospital or go out to eat. In case of any respiratory symptoms such as fever and dry cough, the companions are guided to the fever clinic of our hospital. Symptomatic companions are not allowed to continue the accompanying at the ward. Health education on COVID-19 prevention and control is also strengthened for the companions. Instructions are provided for companions on personal protective measures. The companions are not allowed to go in and out of the workplace in the word and leave the ward at will. Neither are the companions permitted to go to other words or gather together and chat.

c) Training and appraisal of all workers and medical staff are continuously carried out. All workers and medical staff are educated to raise their awareness 
that everybody is a practitioner of infection control. The training system for all workers and medical staff on epidemic prevention and control is executed. The objects of training should include all medical staff, administrative staff, security personnel, logistics (including those in the outsourced services), and companions. The training contents are varied for different posts, and the personnel is appraised after training. All workers and medical staff are required to participate in the test about the knowledge related to COVID-19 prevention and control, which is held on the COVID-19 learning and directed towards medical staff in Guangdong province. Appraisal of medical workers' mastery is shown below in Table 1.

\subsection{Putting the Epidemic Prevention and Control Measures into Effect}

1) Perfecting the pre-examination and triage process and fulfilling the role as a "lookout". At the entrance of the hospital, the epidemic prevention and control strategy of "three checks plus one inquiry" is implemented. All people are required to show their health codes, have their body temperature taken, and the correctness of mask wearing checked. Patients with respiratory symptoms such as fever and/cough are inquired of the clinical manifestations and epidemiological history. All emergency patients and their relatives entering the hospital have their body temperature taken and their epidemiological history registered. All inpatients are required to have proof of a negative result of the PCR COVID-19 test valid for 7 days and admitted via the outpatient and emergency clinic. Patients with fever are guided to seek medical care at the fever clinic. The new inpatients and their relatives must have their body temperature taken and epidemiological history inquired at the ward entrance. They are required to sign the commitment letter for the COVID-19 screening. Admission to the ward is not allowed unless with proof of a negative result of the PCR COVID-19 test.

Table 1. Appraisal of medical workers' mastery of the knowledge related to COVID-19 prevention and control in Guangdong Province in 2021.

\begin{tabular}{|c|c|c|c|c|c|}
\hline $\begin{array}{l}\text { Ranking of the cities in } \\
\text { terms of the number of } \\
\text { participants in the } \\
\text { appraisal }\end{array}$ & $\begin{array}{l}\text { Number of } \\
\text { participants } \\
\text { scoring } 100 \\
\text { points }\end{array}$ & $\begin{array}{l}\text { Ranking of the districts } \\
\text { in terms of the number } \\
\text { of participants in } \\
\text { the appraisal }\end{array}$ & $\begin{array}{l}\text { Number of } \\
\text { participants } \\
\text { scoring } 100 \\
\text { points }\end{array}$ & $\begin{array}{l}\text { Ranking of the affiliations } \\
\text { in terms of the number of } \\
\text { participants in the } \\
\text { appraisal }\end{array}$ & $\begin{array}{l}\text { Number of } \\
\text { participants } \\
\text { scoring } 100 \text { points }\end{array}$ \\
\hline \multirow[t]{4}{*}{ Guangzhou City } & 179,740 & Yuexiu District & 42,855 & $\begin{array}{l}\text { Third Affiliated Hospital of } \\
\text { Sun Yat-sen University }\end{array}$ & 2376 \\
\hline & & Baiyun District & 27,107 & $\begin{array}{l}\text { Guangzhou Women and } \\
\text { Children's Medical Center }\end{array}$ & 2344 \\
\hline & & Tianhe District & 23,699 & $\begin{array}{l}\text { First Affiliated Hospital of } \\
\text { Jinan University }\end{array}$ & 2274 \\
\hline & & Haizhu District & 21,233 & $\begin{array}{l}\text { Sixth Affiliated Hospital of } \\
\text { Sun Yat-sen University }\end{array}$ & 2118 \\
\hline Shenzhen City & 92,481 & & & & \\
\hline Foshan City & 61,996 & & & & \\
\hline
\end{tabular}


2) Supervision and inspection of normalizing the COVID-19 prevention and control work

a) The infection department builds a team of infection control supervisors. This team is composed of the head nurse, infection control nurses, and infection control doctors. The name list is filed with the infection department of the hospital. The infection control supervisors at the infection department must participate in the department-level training and the test of the supervisors organized by the hospital. The supervision checklist for normalized COVID-19 prevention and control is administered to the supervision or inspection work at least once per week. Any problems discovered are analyzed and corrected. These problems are timely reported to the infection management department of the hospital for joint discussion, analysis, and looking for redress. The department director is the first responsible person for COVID-19 prevent and control of the infection department. He or she is responsible for organizing self-inspection, conference, learning, and continuous improvement. The department director is also the person who signs and makes records of the above work.

b) The hospital infection control department organizes a team of supervisors responsible for infection control at the hospital, making regular rounds of inspection at key departments and the entire hospital. The hospital infection control department leads the regular joint supervision and inspection across the departments. The purpose is to identify the hidden risks, establish a problem ledger, and put the corrective measures into effect with the items that have been corrected crossed out. The conference is held regularly in the hospital infection management committee to discuss the supervision and inspection of epidemic prevention and control and the correction situation. Timely solutions are sought for the problems upon discovery. The supervision and inspection results are included as part of the performance appraisal of the department and the personnel.

c) Disinfection management of the environment of care is improved according to relevant operational guidelines. Ventilation is strengthened. Air disinfection machine is used for populated places. Ultraviolet irradiation is adopted where no people come and go. Special attention is paid to the scope and duration of ultraviolet irradiation. Disinfection of object surfaces and floor requires special attention. High-frequency contact surfaces, such as doors, should be disinfected more frequently. Respiratory secretions, excretions, and vomitus from the patients are handled in strict accordance with the requirements. Medical waste management and terminal disinfection are also performed according to the rules. Two rooms are reserved as transition ward for admitting patients whose nucleic acid test results have not come out yet or for screening suspected patients. Patients waiting for the nucleic acid test to come out are kept separately [4].

\section{Result}

\subsection{An Overview of the Screening}

From March 1, 2020 to March 31, 2021 three ward areas of the infection de- 
partment included 3056 patients and 868 companions. All of the inpatients and their companions were required to offer proof of a negative result of the PCR COVID-19 test valid for 7 days. There were 265 inpatients with fever and 113 inpatients with respiratory symptoms. They were timely quarantined and received repeated nucleic acid tests, blood tests, and chest X-rays, all of which precluded COVID-19. None of the inpatients, companions, and medical staff was infected with COVID-19.

\subsection{Screening Results}

The participation rate of the test about COVID-19 prevention and control was $100 \%$ among all medical staff, including 83 doctors and 114 nurses. The knowledge mastery rate was also $100 \%$.

\section{Discussion}

Our hospital is a designated comprehensive hospital in Guangdong Province for COVID-19 treatment. Our hospital has an independent fever clinic and an isolation ward. The infection department is a department where COVID-19 epidemic prevention and control are particularly important and challenging due to the large number of patient visits, complex disease constitution, high patient turnover, and high population mobility. For the infection department, normalizing the COVID-19 epidemic prevention and control work is of special importance. China has successfully tamed the epidemic so far, though the situation seems to worsen overseas. China is still facing pressure from COVID-19 imported from abroad. Hence, the epidemic risk lingers on. As an emerging infectious disease, COVID-19 carries enormous uncertainties. The " $3+1$ " mode for epidemic prevention and control is conducted at our hospital, a designated comprehensive hospital for COVID-19 treatment in Guangdong. Continuous efforts have been made to perfect the epidemic prevention and control system, enhance patient, companion and medical staff management, put the preventive measures into effect, and strictly carry out the COVID-19 screening procedure. The majority of the high-risk population can be detected by COVID-19 screening at the three entrances (hospital entrance, entrance to the emergency and outpatient clinic, and entrance to the ward). The overall difficulty of COVID-19 prevention and control is lowered by combining the screening strategy at the three entrances with the epidemic prevention and control at the ward. Improving the epidemic prevention and control system at the ward offers a robust institutional guarantee for implementing the anti-COVID-19 measures at the ward and helps standardize the preventive measures. The " $3+1$ " mode for epidemic prevention and control requires coordination across departments and sites. The actual situation of the hospital and the management status quo of the ward area deserve our attention during the implementation of the " $3+1$ " mode. We should highlight passage management, and timely adjust the number of available passages and the configuration of the COVID-19 screening personnel according to the population 
flow to avoid overcrowding and aggregation. COVID-19 screening quality and personnel management should be simultaneously strengthened to minimize the risk of COVID-19 infection.

\section{Funding}

This work was funded by the Scientific Research Fund for Nursing at the Third Affiliated Hospital of Sun Yat-sen University (2019HL10).

\section{Conflicts of Interest}

The authors declare no conflicts of interest regarding the publication of this paper.

\section{References}

[1] Tang, X.P. (2020) Guangzhou's Interconnected Reform of Medical Care, Medical Insurance and Pharmaceutical Industry Ensures the High Efficiency and Orderliness of COVID-19 Treatment. Chinese Journal of Health Policy, 2020, 93-94.

[2] The COVID-19 Diagnosis and Treatment Plan (Trial Version 6) (2020) Chinese Journal of Infection Control, 19, 192-195.

[3] Liu, X.W., Chi, L.W., Qu, Y.M., et al. (2021) Application of Grid Management in COVID-19 Prevention and Control. Modern Hospitals, 21, 264-266.

[4] Zhu, Y.M., Liu, C.N. and Liu, Y. (2021) Management of the Inpatients and Their Companions at the Ordinary Ward during the COVID-19 Epidemic. Journal of Qilu Nursing, 27, 161-163.

[5] Fei, X.Q. and Guo, H. (2021) Comprehensive Prevention and Control Strategies for Large-Scale Healthcare Institutions during the COVID-19 Epidemic and the Appraisal. Journal of Preventive Medicine Information, 37, 14-19.

[6] Gu, W., Xue, J.J., Chen, H.Y., et al. (2021) Prevention and Control Measures at a Cadre Ward of a Hospital during the COVID-19 Epidemic. Journal of Navy Medicine, 42, 50-51. 